\title{
A Percepção de Qualidade de Vida de Pessoas Portadoras de Deficiência Física Pode ser Influenciada Pela Prática de Atividade Física?
}

\section{Can the Self-Perceived Quality of Life of People with Special Needs be Influenced by Practice of Physical Activity?}

Franco Noce ${ }^{1,2}$

Mário Antônio de Moura Simim

Marco Túlio de Mello²

1. Laboratório de Psicologia do Esporte - Centro Universitário de Belo Horizonte-Uni-BH.

2. Centro de Estudos de

Psicobiologia e Exercício,

Departamento de Psicobiologia

- EPM/Unifesp.

Endereço para correspondência:

Franco Noce

Rua dos Construtores, 464 - Alípio de Melo.

30830-550 - Belo Horizonte, MG.

E-mail: fnoce@psicobio.epm.br

Submetido em 31/10/2007

Versão final recebida em 29/04/2008 Aceito em 28/11/2008

\begin{abstract}
RESUMO
A prática regular de atividade física promove uma série de benefícios que vão além da esfera física. A qualidade de vida, segundo diversas fontes, diz respeito ao grau de satisfação de um indivíduo com os múltiplos aspectos da sua vida. As pessoas portadoras de deficiência física podem apresentar níveis de sedentarismo elevados, influenciando de forma decisiva na percepção de sua qualidade de vida. Assim, o objetivo deste estudo foi verificar o efeito de 12 semanas de prática de atividade física na percepção do nível de qualidade de vida de deficientes físicos. Participaram do estudo 20 deficientes físicos, do gênero masculino, divididos em dois grupos, Sedentários e Ativos. Como instrumento de estudo foi utilizado um questionário de dados demográficos e o World Health Organization Quality of Life (WHOQOL-Bref). Em geral, a média de idade dos voluntários era de 38,1 anos, possuíam o $1^{\circ}$ grau incompleto, moradia própria, eram solteiros e predominantemente portadores de poliomielite. O grupo Ativo apresentou escores mais elevados na qualidade de vida em todas as dimensões (física, psicológica, social e ambiental) do instrumento. Os resultados obtidos com o presente estudo comprovaram que o nível de qualidade de vida dos deficientes físicos Ativos foi melhor do que a dos Sedentários.
\end{abstract}

Palavras-chave: qualidade de vida, atividade física, deficiência física.

\begin{abstract}
The positive effects of regular physical activity practice are not restricted to physical well being. Quality of life regards the overall sense of well-being of an individual in relation to the multiple aspects of his/her life. People with special needs may present high levels of sedentarism, which has a remarkable influence on their self-perceived quality of life. Therefore, the objective of the present study was to assess the effect of 12 weeks of physical activity practice on the perception of the quality of life of individuals with special needs. 20 male individuals with physical disabilities participated in the study. They were divided into two groups, Sedentary and Active. The instruments used were a questionnaire on demographic data and the World Health Organization Quality of Life (WHOQOL-Brief). Mean age of the volunteers was 38.1 years, they were single, had not finished elementary school and lived in their own house. Most of them had poliomyelitis. The Active group presented higher scores in all domains of the instrument (physical, psychological, social and environmental). The results obtained in the present study showed that the quality of life of Active people with special needs was better than that of the Sedentary ones. Consequently, we strongly recommend that people with physical disabilities be encouraged to participate in physical and/or sports activity programs regularly.
\end{abstract}

Keywords: quality of life, physical activity, physical disability.

\section{INTRODUÇÃO}

Sabe-se que a atividade desportiva praticada por pessoas portadoras de deficiência existe desde a Grécia ntiga. No entanto, foi somente após a Segunda Guerra Mundial que o desporto adaptado para os deficientes teve maior avanço no contexto da prevenção e da reabilitação física, social e psíquica ${ }^{(1,2)}$. De modo geral, os portadores de deficiência física ou de qualquer outra deficiência obtêm efeitos positivos para a saúde física, mental e social pela prática regular de atividades de lazer ou desportivas adaptadas, tenham estas finalidades competitivas ou não(1), embora se saiba que sua participação em atividades físicas regulares seja inferior à da do restante da população(3).

De forma geral, segundo Haddad et al.(4) e Silva ${ }^{(5)}$, os deficientes físicos, da mesma maneira que população em geral, alteram os seus hábitos de vida, tornando-se mais sedentários. Essa condição implica, além de uma série de problemas (p. ex.: intolerância à glicose; maior prevalência de aterosclerose), normalmente, aumento da obesidade, tornando o indivíduo mais suscetível às diversas enfermidades. Mello 
et al. (6) observaram que, na maioria da vezes, os deficientes físicos iniciantes de um programa de atividade física têm como características depressão e distúrbios do sono.

Diversos estudos têm evidenciado a importância da prática regular de atividade física como meio de prevenir doenças associadas ao sedentarismo ${ }^{(3)}$. De acordo com Labronici et al. ${ }^{(7)}$, a prática esportiva para o portador de deficiência é um mecanismo facilitador da inclusão na sociedade.

Zuchetto e Castro(8) ressaltam a importância do apoio da família e dos amigos para auxiliar o deficiente a adotar um estilo de vida mais ativo. Fox et al. ${ }^{(9)}$ destacam a influência da atividade física no desenvolvimento biopsicossocial, possibilitando a melhora da saúde e qualidade de vida do praticante.

A qualidade de vida tem sido cada vez mais investigada; diversos fatores podem ser associados a ela ${ }^{(10)}$ (figura 1). A atividade física, nesse contexto, se apresenta como um indicador potencial e diversos estudos têm demonstrado isso.

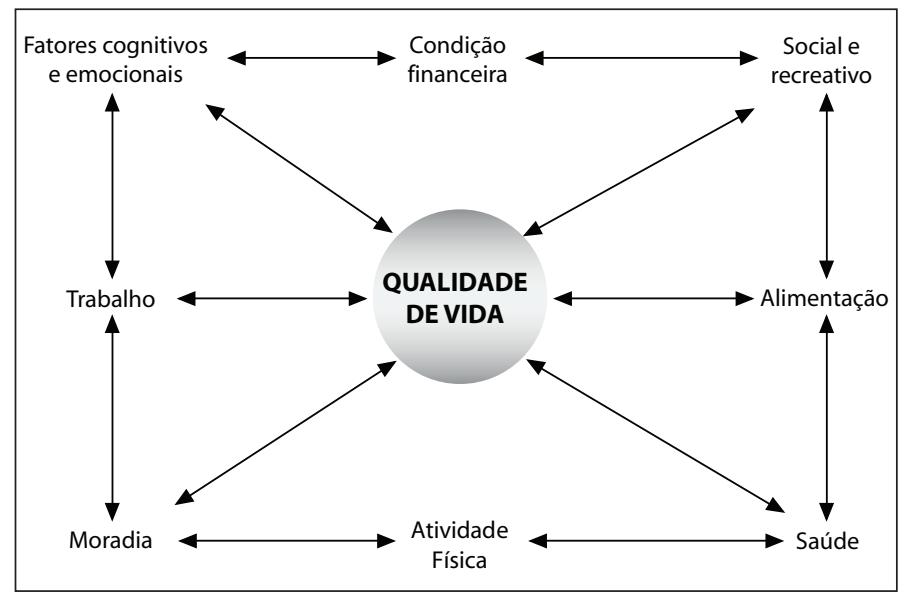

Figura 1. Fatores que afetam a qualidade de vida (Spirduso10)

Samulski e Noce ${ }^{(11)}$ destacaram que, quando se fala sobre o ser humano, devem-se ter em mente suas relações biopsíquicas e sociais, levando-se em conta sua saúde mental e sua qualidade de vida. Seria necessário haver estudos específicos com as pessoas portadoras de deficiência, sobre as condições que permitem boa qualidade de vida, bem como a criação de alternativas de intervenção que vise seu bemestar biopsicossocial.

Dessa forma, o objetivo deste estudo foi o de verificar a percepção de qualidade de vida de deficientes físicos ativos e sedentários.

\section{MÉTODOS}

Participaram do estudo 20 indivíduos do gênero masculino que apresentavam uma deficiência física, sem comprometimentos cognitivos, divididos em dois grupos, (1) o Sedentário e (2) o Ativo. O primeiro foi constituído por 10 indivíduos que não praticavam nenhuma atividade esportiva havia no mínimo seis meses. Já o segundo grupo foi composto por 10 atletas de basquetebol em cadeira de rodas.

Foram utilizados como instrumentos de pesquisa: o Questionário de Dados Demográficos e o WHOQOL-bref (12).

O questionário de Dados Demográficos é composto por 10 questões, cuja finalidade é a identificação do perfil da amostra. As questões abordam aspectos relativos aos dados pessoais (idade, estado civil, moradia, remuneração, escolaridade, nutrição); deficiência (tipo, quando foi adquirida) e atividade física (tempo de prática, tipo de atividades, motivos atuais para a prática esportiva, motivos de abandono da prática esportiva).
A qualidade de vida foi mensurada utilizando-se o questionário World Health Organization Quality of Life Instruments (WHOQOL_bref), elaborado pela Organização Mundial de Saúde ${ }^{(13)}$, validado para a população brasileira por Fleck et al.(12). O questionário WHOQOL_bref, formado por 26 questões, é multidimensional e avalia a qualidade de vida geral (dois itens), assim como esta em quatro domínios (24 itens): físico, psicológico, social e ambiental.

O processo da coleta de dados do instrumento foi realizado a cada 30 dias, durante 12 semanas, em locais e dias distintos. As coletas referentes ao grupo Sedentário foram realizadas na União dos Paraplégicos de Belo Horizonte (Unipabe), que é uma entidade filantrópica prestadora de serviços comunitários para vários deficientes físicos. As do grupo Ativo foram realizadas na quadra do Corpo de Bombeiros, antes do treinamento da equipe de basquetebol em cadeiras de rodas.

Para a análise dos dados utilizou-se estatística descritiva composta por média, desvio padrão e distribuição de frequência, e para se fazer a comparação entre os grupos Ativo e Sedentário, foi realizado o teste t independente. Já para comparar os períodos de coleta foi utilizada análise de variância (Anova one-way), adotando-se o valor de $p \leq 0,05$ para identificar as diferenças em todas as comparações. Todos os procedimentos foram tabulados e analisados no pacote estatístico SPSS for Windows ${ }^{\circledast}$, versão 11.0.

Todos os indivíduos envolvidos na pesquisa foram informados sobre os objetivos da mesma e, necessariamente, assinaram um termo de consentimento para a efetiva participação. A identidade dos participantes foi resguardada.

A pesquisa foi conduzida de acordo com as normas e diretrizes do Comitê de Ética do Centro Universitário de Belo Horizonte - CEP-Uni$\mathrm{BH}$, respeitando-se também as normas estabelecidas pelo Conselho Nacional de Saúde (Resolução CNS 196/96).

\section{RESULTADOS}

Os resultados são apresentados seguindo a ordem dos procedimentos metodológicos adotados, com o intuito de facilitar sua compreensão, pelo que se optou por exibir a seguinte sequência lógica de eventos: (1) caracterização da amostra; (2) análise da qualidade de vida por domínio.

\section{Caracterização da amostra}

A média de idade dos voluntários foi de 38,10 anos ( $\pm 11,73$ ); não foi encontrada diferença significativa entre as idades nos dois grupos. É importante destacar que o grupo Ativo apresentou melhor nível educacional em relação ao Sedentário, além de ter apresentado maior independência (tabela 1).

Tabela 1. Dados demográficos da amostra

\begin{tabular}{|c|c|c|c|}
\hline Variáveis & Geral & Ativos & Sedentários \\
\hline Idade & $38,10( \pm 11,73)$ & $33,10( \pm 8,76)$ & $43,10( \pm 12,58)$ \\
\hline Escolaridade & $\begin{array}{l}1^{\circ} \mathrm{g} \cdot \text { incompleto }-60 \% \\
1^{\circ} \mathrm{g} \cdot \text { completo }-10 \% \\
2^{\circ} \mathrm{g} \cdot \text { completo }-25 \% \\
3^{\circ} \mathrm{g} \cdot \text { incompleto }-5 \%\end{array}$ & $\begin{array}{l}1^{\circ} \mathrm{g} \cdot \text { incompleto }-30 \% \\
1^{\circ} \mathrm{g} \cdot \text { completo }-20 \% \\
2^{\circ} \mathrm{g} \cdot \text { completo }-40 \% \\
3^{\circ} \mathrm{g} \cdot \text { incompleto }-10 \%\end{array}$ & $\begin{array}{l}1^{\circ} \mathrm{g} \text {. incompleto }-90 \% \\
2^{\circ} \mathrm{g} \text {. completo - } 10 \%\end{array}$ \\
\hline Trabalho & $\begin{array}{l}\text { Trabalham - 65\% } \\
\text { Não trabalham - 35\% }\end{array}$ & $\begin{array}{l}\text { Trabalham - 70\% } \\
\text { Não trabalham - 30\% }\end{array}$ & Não trabalham - 100\% \\
\hline Moradia & $\begin{array}{l}\text { Própria - 55\% } \\
\text { Alugada - 10\% } \\
\text { Vive com parentes - 35\% }\end{array}$ & $\begin{array}{l}\text { Própria - } 60 \% \\
\text { Vive com parentes - 40\% }\end{array}$ & $\begin{array}{l}\text { Própria - 50\% } \\
\text { Alugada - } 20 \% \\
\text { Vive com parentes - 30\% }\end{array}$ \\
\hline Estado civil & $\begin{array}{l}\text { Solteiro - 55\% } \\
\text { Casado - 40\% } \\
\text { Viúvo - 5\% }\end{array}$ & $\begin{array}{l}\text { Solteiro - } 40 \% \\
\text { Casado - 50\% } \\
\text { Viúvo - 10\% }\end{array}$ & $\begin{array}{l}\text { Solteiro - 70\% } \\
\text { Casado - 30\% }\end{array}$ \\
\hline
\end{tabular}


A tabela 2 apresenta os resultados relativos ao tipo de deficiência e ao período de sua aquisição. Verificou-se que a poliomielite foi o tipo de deficiência física predominante, afetando quase a metade (45\%) dos indivíduos pesquisados. Além disso, 85\% dos indivíduos adquiriram a doença no período pós-natal.

Tabela 2. Distribuição da amostra segundo o tipo de deficiência física e o período em que esta foi adquirida

\begin{tabular}{l|c}
\hline \multicolumn{1}{c|}{ Variáveis } & $\%$ \\
\hline \multirow{2}{*}{ Tipo de deficiência } & Amputação $-5 \%$ \\
& Poliomelite $-45 \%$ \\
& Lesão Medular $-25 \%$ \\
& Paralisia Cerebral $-5 \%$ \\
& Acidente Vascular Cerebral (AVC) - 5\% \\
Deficiência Física Adquirida $-15 \%$
\end{tabular}

\section{Análise do índice da qualidade de vida por dimensões}

Nos escores relacionados ao domínio físico, apresentados no gráfico 1, verifica-se que o grupo Ativo apresentou valores significativamente superiores aos do Sedentário. Observou-se declínio acentuado nos escores do grupo Sedentário entre a primeira e segunda coletas e entre a primeira e terceira coletas. Apesar disso, não foram encontradas diferenças significativas entre essas mesmas coletas. Também se pode observar a estabilidade dos escores no grupo Ativo.

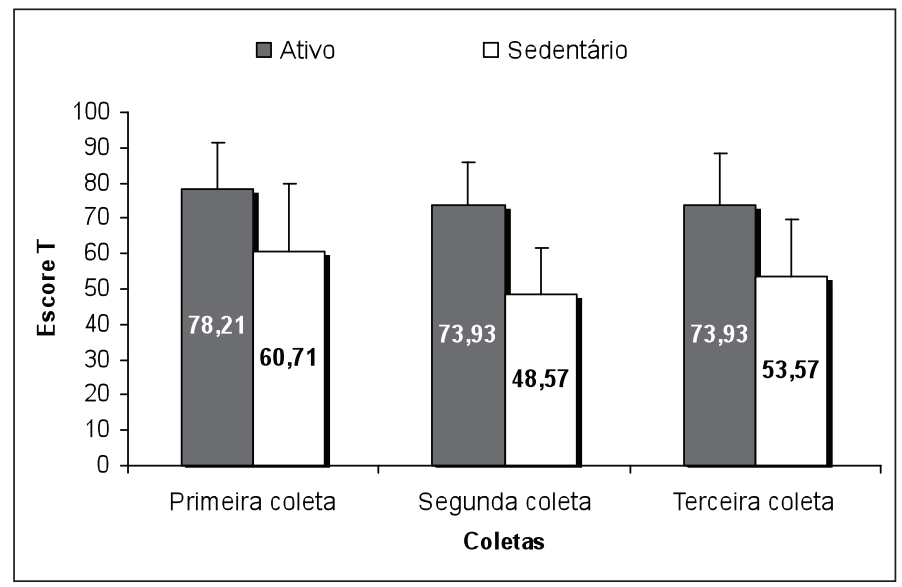

Gráfico 1. Média geral dos escores referentes ao domínio físico $\left.{ }^{*} p \leq 0,05{ }^{* *} p \leq 0,01\right)$

Observando-se o gráfico 2, percebe-se que os valores dos escores do "grupo Ativo", envolvendo o domínio psicológico, foram superiores aos valores do Sedentário em todas as coletas $(p=0,000)$. Não foram encontradas diferenças significativas entre as coletas em ambos os grupos.

O gráfico 3 mostra que, apesar de os valores dos escores (relacionados ao domínio social) do grupo Ativo serem superiores aos do Sedentário em todas as coletas, não foram significativos. Também não foram encontradas diferenças significativas entre eles em cada uma das coletas.

Nas duas últimas coletas ( $p \leq 0,05)$, em relação ao domínio Ambiental (gráfico 4), verificou-se que a percepção da qualidade de vida do grupo Ativo foi superior à do Sedentário. Mais uma vez, ao longo das coletas, também se pode observar a estabilidade dos escores nos dois grupos.

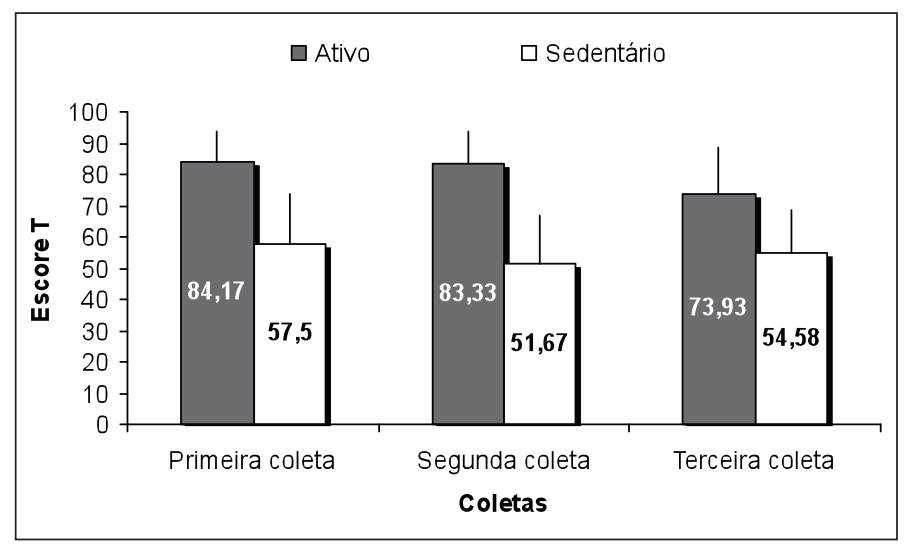

Gráfico 2. Média geral dos escores referentes ao domínio psicológico $\left({ }^{*} p \leq 0,05\right.$; $* * p \leq 0,01)$

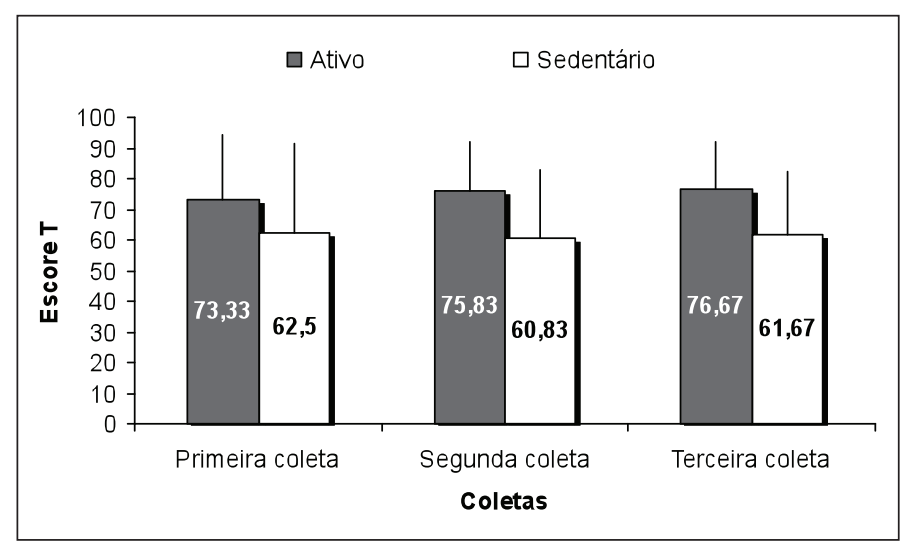

Gráfico 3. Média geral dos escores referentes ao domínio social ( $\left.{ }^{*} p \leq 0,05 ;{ }^{* *} p \leq 0,01\right)$

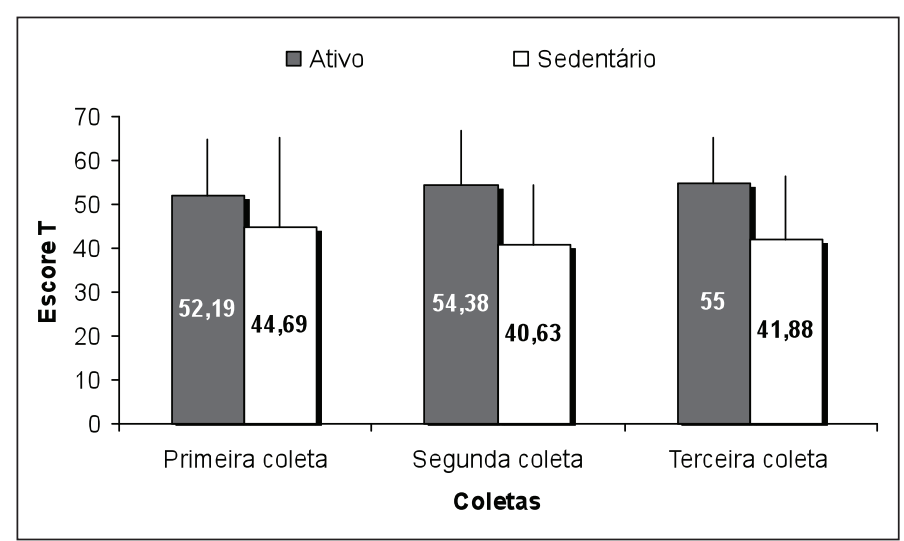

Gráfico 4. Média geral dos escores referentes ao domínio ambiental $\left({ }^{*} p \leq 0,05 ;{ }^{* *} p \leq 0,01\right)$

Observa-se que, de acordo com o gráfico 5, pode-se observar que o domínio que apresentou maior escore para o grupo Ativo foi o Psicológico. Já para o grupo Sedentário, foi o Social. Em todos os domínios foram verificadas diferenças significativas entre os dois grupos.

\section{DISCUSSÃO}

A idade média da amostra em nosso estudo ficou compreendida em uma faixa etária potencialmente produtiva ${ }^{(14)}$. Segundo Singer et al. ${ }^{(15)}$, com o avanço da idade observam-se declínio da capacidade funcional do indivíduo e manutenção da dimensão saúde mental. No entanto, não há consenso nos resultados das pesquisas sobre a influência da idade na qualidade de vida. Diante de nossa constatação de que a idade compromete mais marcantemente as dimensões do componente físico, nosso trabalho corrobora os estudos de Santos ${ }^{(14)}$ e Singer et al. ${ }^{(15)}$. 


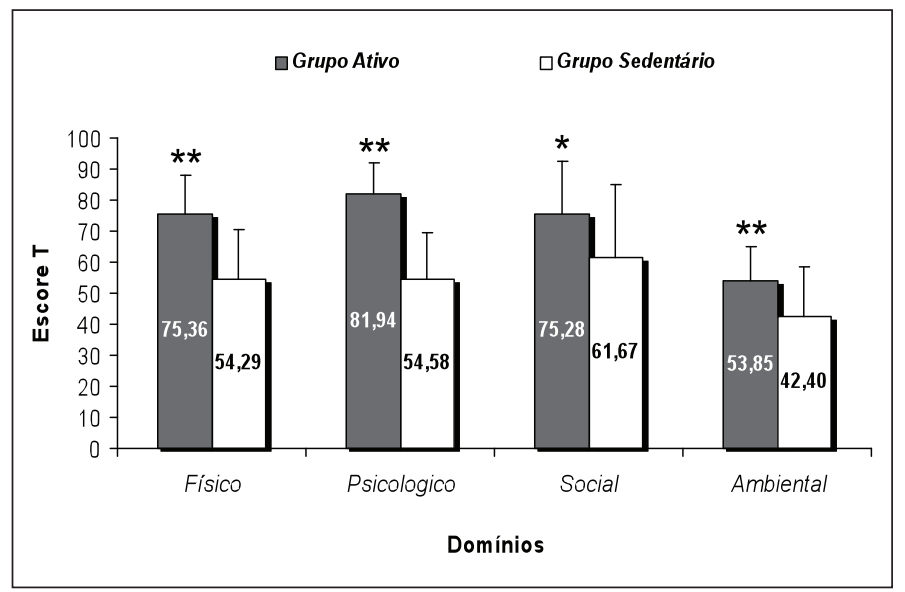

Gráfico 5. Escore geral dos domínios por grupo $\left({ }^{*} p \leq 0,05\right.$; $\left.{ }^{* *} p \leq 0,01\right)$

A incidência da poliomielite em uma parcela significativa dos pesquisados pode ter relação direta com sua média de idade $(38,1$ anos $\pm 11,73)$, a qual não era muito baixa. Essa relação surgiu devido ao fato de que, algumas décadas atrás, não eram realizadas no Brasil campanhas regulares de vacinação contra a pólio, o que provocava o aumento dos casos dessa doença no período pós-natal ${ }^{(8)}$. Os fatores determinantes causadores das deficiências após esse período advinham das encefalites, das meningites, dos acidentes e das doenças adquiridas, conforme verificaram Zuchetto e Castro ${ }^{(8)} \mathrm{em}$ seu estudo, em que $72 \%$ da amostra adquiriram a deficiência no período pós-natal.

De acordo com Silva ${ }^{(16)}$, a obtenção da qualidade de vida relaciona-se com o estilo de vida da pessoa, sua moradia, sua forma de lidar com o trabalho e a segurança financeira proporcionada por si própria. O grau de escolaridade ou nível de instrução têm relação íntima com a inserção no mercado de trabalho. Espera-se que, quanto maior o nível de escolaridade, melhor o posto de trabalho ocupado e, consequentemente, melhor a renda familiar. O nível de escolaridade apresentado por quase todos os indivíduos do grupo sedentário é muito baixo, fator que acabou influenciando suas oportunidades de emprego. Assim, os benefícios sociais acabam sendo de grande valia, principalmente para o grupo Sedentário, já que boa parte do grupo Ativo dispõe de renda própria.

Em relação à qualidade de vida, os diversos trabalhos publicados até aos dias de hoje referiram-se às propriedades psicométricas do WHOQOL ${ }^{(13,17-22)}$, bem como à utilização do mesmo em amostras de pacientes psiquiátricos ou em estado de internação ${ }^{(23-27)}$ e na população idosa(28-30).

O Brasil é um país onde parcela significativa da população (14,5\%) é portadora de algum tipo de deficiência ${ }^{(31)}$. Entretanto, pouco se tem estudado a respeito da qualidade de vida e dos fatores associados nessa população, o que evidencia ainda mais a importância desse trabalho.

No que se refere aos escores da qualidade de vida, eles são uma escala positiva, pois quanto maior o escore, melhor a qualidade de vida ${ }^{(12)}$. Pereira et al.(32) evidenciaram que alterações em um ou mais domínios podiam implicar alterações na qualidade de vida.

Os resultados deste estudo indicaram escores mais elevados de qualidade de vida para o grupo Ativo. Diversos autores ${ }^{(1,6,8-9,33)}$ já demonstraram que a prática de atividade física proporciona benefícios biopsicossociais ao indivíduo.

Nunes e Freire ${ }^{(34)}$, em um estudo envolvendo cirugiões-dentistas ( $n=237)$ da cidade de Goiânia, verificaram a existência de esco- re igual a 70,3 $( \pm 14,6)$ para o domínio Físico. Este, já no estudo de Roque e Forones ${ }^{(35)}$ com 45 pacientes com câncer colorretal, no segundo ciclo de quimioterapia em tratamento adjuvante, apresentou, respectivamente, escores iguais a $69( \pm 6), 68( \pm 6)$ e $67( \pm 7)$ para as três coletas. Em ambos, os estudos, o domínio Físico obteve escores mais elevados.

Os deficientes físicos sedentários deveriam ser estimulados a participar de um programa de atividade física, vista a enorme importância que a prática regular de atividade física tem para a saúde das pessoas, pois ela reduz a possibilidade direta dos riscos do desenvolvimento precoce das doenças crônico-degenerativas e da mortalidade ${ }^{(5,33)}$.

Já no que diz respeito aos deficientes físicos ativos, a prática orientada de atividades físicas ou esportivas promove vários benefícios orgânicos relacionados com os aspectos metabólicos, músculo-osteoarticular e cardiorrespiratório ${ }^{(8)}$. Acredita-se que o estilo de vida ativo possa promover maiores benefícios relacionados com a saúde física.

Para o portador de deficiência física que se envolve constantemente em atividades esportivas, ocorre "sensação" de estar vivendo uma vida mais saudável, percepção de possuir melhor imagem corporal e o reforço de sua auto-estima; a vida the fazendo mais sentido ${ }^{(8)}$. Esses benefícios psicológicos conseguidos por influência da prática regular de atividades físico-esportivas se refletem, de modo geral, nas relações de trabalho, na vida afetiva e social. Contudo, Samulski e Noce ${ }^{(36)}$ apontam existir, como resultado da prática de exercícios físicos, redução dos níveis da ansiedade, do estresse e da depressão; melhora no humor; aumento do bem-estar físico e psicológico; melhor funcionamento orgânico geral e disposição física e mental aumentada.

Segundo Rabelo e Néri(37), os recursos psicológicos como o otimismo, o controle pessoal e o senso de significado são importantes, como sendo reservas que permitem às pessoas enfrentar melhor os eventos críticos da vida. Diversos estudos ${ }^{(32,37)}$ verificaram que o bem-estar psicológico pode promover comportamentos saudáveis, uma vez que pessoas dotadas de senso de autovalor acreditam em seu poder de controle e são otimistas quanto a seu futuro, além de mais propensas a adotar hábitos mais saudáveis e conscientes.

Em relação ao domínio Social, os resultados de nosso estudo corroboraram os encontrados por Paskulin e Molzahnn ${ }^{(38)}$. Esses autores realizaram um estudo para analisar os fatores que contribuíam para a qualidade de vida de idosos no Canadá $(n=202)$ e no Brasil ( $n=288$ ), tendo encontrado, na amostra canadense, escores mais elevados para a qualidade de vida nos domínios físico, psicológico e ambiental. Porém, corroborando nosso estudo, para o domínio social não foram encontradas diferenças significativas entre os dois países. Os autores verificaram existir o mesmo padrão de fatores (satisfação com a saúde, dinheiro suficiente e oportunidades para atividades de lazer) como sendo os que mais contribuem para a mudança da qualidade de vida. Saliente-se que, de acordo como Araújo e Araújo ${ }^{(33)}$, uma vida voltada para a prática de exercícios físicos ou esportivos pode melhorar também os aspectos relacionados com o convívio social.

De fato, observa-se na literatura atual que os efeitos agudos em uma sessão de exercícios físicos estão inversamente relacionados com a incidência de doenças cardiovasculares e obesidade, assim como diversos benefícios psicológicos ${ }^{(39)}$. Isso sugere que o exercício pode ser uma estratégia valiosa em curto prazo para a autorregulação do humor em situações da vida diária. 
Conclui-se que a prática regular de atividade física parece ter efeitos positivos na percepção de bons níveis de qualidade de vida. A constatação dos escores superiores, nos diferentes domínios do instrumento utilizado, demonstra que o nível da qualidade de vida dos deficientes físicos ativos é melhor do que o dos sedentários.

Contudo, também é importante destacar que algumas outras variáveis, como o trabalho-renda, o nível de instrução e a moradia, podem ter contribuído para melhor nível da qualidade de vida a favor dos deficientes físicos ativos. Assim, destaca-se a necessidade da realização de novos estudos, com o intuito de verificar que outros fatores, além dos já verificados, podem interferir na qualidade de vida do deficiente físico. Estudos longitudinais, acompanhando o efeito da prática de atividade física por um período mais prolongado, são importantes para investigar se as modificações ao longo do tempo das variáveis acima destacadas podem contribuir para a qualidade de vida do individuo.

\section{AGRADECIMENTOS}

Ao Centro Universitário de Belo Horizonte (UNIBH), Associação Fundo de Incentivo à Psicofarmacologia (Afip), Centro de Estudos em Psicobiologia e Exercício (Cepe), Fapesp (03/06297-3, Cepid 98/143033), Fada, CNPQ e INAPSS.

Todos os autores declararam não haver qualquer potencial conflito de interesses referente a este artigo.

\section{REFERÊNCIAS BIBLIOGRÁFICAS}

1. Souza P. O Esporte na Paraplegia e Tetraplegia. Rio de Janeiro: Guanabara Koogan, 1994.

2. Araújo P. Desporto Adaptado no Brasil: Origem, Institucionalização e Atualidade. Braślia: Ministério da Educação e do Desporto/INDESP, 1998.

3. Heath GW, Fentem PH. Physical activity among persons with disabilities: A public health perspective. Exerc Sport Sci Rev 1997;25:195-234

4. Haddad S, Silva PRS, Pereira Barretto AC, Ferraretto I. The effect of short term Aerobic physical training using upper limbs in paraplegic persons with mild to moderate hypertension. Arq Bras Cardiol 1997;69:169-73.

5. Silva A. Atleta Portador de Deficiência. In: Ghorayeb N, Barros T, editors. O Exercício: Preparação Fisiológica, Avaliação Médica, Aspectos Especiais e Preventivos. São Paulo: Atheneu, 1999b;321-36.

6. Mello MT, Natal CL, Cunha JM, Tufik S. Epidemiologia do padrão de sono em adultos desportistas portadores de lesão medular. Rev Port Med Desp 1995;13:89-100.

7. Labronici RHDD, Cunha MCB, Oliveira ASB, Gabbai AA. Sport as integration factor of the physically handicapped in our society. Arq Neuropsiquiatr 2000;58:1092-9.

8. Zuchetto A, Castro R. As Contribuições das Atividades Físicas para a Qualidade de Vida dos Deficientes Físicos. Kinesis 2002;26:52-68.

9. Fox KR, Boutcher SH, Faulkner GE, Biddle SJH. The case for exercise in the promotion of mental health and psychological well-being. In: Biddle SJH, Fox KR, Boutcher SH, editors. Physical Activity and Psychological Well-being. London: Routledge, 2000;1-9.

10. Spirduso WW. Dimensões Físicas do Envelhecimento. Barueri: Manole, 2005.

11. Samulski DM, Noce F. A importância da atividade física para a saúde e qualidade de vida: um estudo entre professores, alunos e funcionários da UFMG. Revista Brasileira Atividade Física Saúde 2000;5:5-21.

12. Fleck MPA, Louzada S, Xavier M, Chachamovich E, Vieira G, Santos L, et al. Aplicação da versão em português do instrumento de qualidade de vida "WHOQOL-bref". Rev Saude Publica 2000;34:178-83.

13. The Whogol Group. Development of the World Health Organization Whogol-Bref Quality of Life Assessment. Psychol Med 1998;28:551-8.

14. Santos, PR. Relação do sexo e da idade com nível de qualidade de vida em renais crônicos hemodialisados. Rev Assoc Med Brás 2006;52:356-9.

15. Singer MA, Hopman WM, MacKenzie TA. Physical functioning and mental health in patients with chronic medical conditions. Qual Life Res 1999:8:687-91.

16. Silva M. Exercício e Qualidade de Vida. In: Ghorayeb N, Barros T, editors. O Exercício: Preparação Fisiológica, Avaliação Médica, Aspectos Especiais e Preventivos. São Paulo: Atheneu 1999a;261-6.

17. Moreno AB, Faerstein E, Werneck GL, Lopes CS, Chor D. Psychometric properties of the World Health Organization Abbreviated Instrument for Quality of Life Assessment in the Pró-Saúde Study. Cad Saúde Publica 2006;22:2585-97

18. Wang WC, Yao G, Tsai YJ, Wang JD, Hsieh CL. Validating, improving reliability, and estimating correlation of the four subscales in the WHOQOL-BREF using multidimensional Rasch analysis. Qual Life Res 2006;15:607-20

19. Ackerman IN, Graves SE, Bennell KL, Osborne RH. Evaluating quality of life in hip and knee replacement: Psychometric properties of the World Health Organization Quality of Life short version instrument. Arthritis Rheum 2006:55:583-90.

20. Chachamovich E, Trentini C, Fleck MP. Assessment of the psychometric performance of the WHOQOL-BREF instrument in a sample of Brazilian older adults. Int Psychogeriatr 2006;27:1-12.
21. Silva Lima AF, Fleck M, Pechansky F, De Boni R, Sukop P. Psychometric properties of the World Health Organization quality of life instrument (WHOQoL-BREF) in alcoholic males: a pilot study. Qual Life Res 2005;14:473-8.

22. Pibernik-Okanović M. Psychometric properties of the World Health Organisation quality of life questionnaire (WHOQOL-100) in diabetic patients in Croatia. Diabetes Res Clin Pract 2001;51:133-43.

23. Akvardar Y, Akdede BB, Ozerdem A, Eser E, Topkaya S, Alptekin K. Assessment of quality of life with the WHOQOL-BREF in a group of Turkish psychiatric patients compared with diabetic and healthy subjects. Psychiatry Clin Neurosci 2006;60:693-9.

24. Sim K, Chan YH, Chua TH, Mahendran R, Chong SA, McGorry P. Physical comorbidity, insight, quality of life and global functioning in first episode schizophrenia: a 24-month, longitudinal outcome study. Schizophr Res 2006;88:82-9.

25. Angermeyer MC, Kilian R, Wilms HU, Wittmund B. Quality of life of spouses of mentally ill people. Int J Soc Psychiatry 2006;52:278-85.

26. Picardi A, Rucci P, De Girolamo G, Santone G, Borsetti G, Morosini P. The quality of life of the mentally ill living in residential facilities: findings from a national survey in Italy. Eur Arch Psychiatry Clin Neurosci 2006;256:372-81.

27. Chand PK, Mattoo SK, Sharan P. Quality of life and its correlates in patients with bipolar disorder stabilized on lithium prophylaxis. Psychiatry Clin Neurosci 2004;58:311-8.

28. Winkler I, Matschinger H, Angermeyer MC, Whogol-Old Group. The Whogol-Old. Psychother Psychosom Med Psychol 2006;56:63-9.

29. Power M, Quinn K, Schmidt S, Whogol-Old Group. Development of the WHOQOL-Old module. Qual Life Res 2005;14:2197-214.

30. Fleck MP, Chachamovich E, Trentini CM. WHOQOL-OLD Project: method and focus group results in Brazil. Rev Saude Publica 2003;37:793-9.

31. Instituto Brasileiro de Geografia e Estatística - IBGE. Nosso Povo: Características da população. Disponível em http://www.ibge.gov.br/7a12/conhecer_brasil/default.php?id_tema_menu=2\&id_tema_submenu=5. Acesso em: 15 março 2008.

32. Pereira RJ, Cota RMM, Franceschini SCC, Ribeiro RCL, Sampaio RF, Priore SE, et al. Contribuição dos domínios físico, social, psicológico e ambiental para a qualidade de vida global de idosos. Revista de Psiquiatria 2006;28:27-38

33. Araújo D, Araújo C. Aptidão Física, Saúde e Qualidade de Vida relacionada à saúde em adultos. Rev Bras Med Esporte 2000;6.

34. Nunes MF, Freire MCM. Qualidade de vida de cirurgiões-dentistas que atuam em um serviço público. Rev Saude Publica 2006;40:1019-26.

35. Roque VMN, Forones NM. Avaliação da qualidade de vida e toxicidades em pacientes com câncer colorretal tratados com quimioterapia adjuvante baseada em fluoropirimidinas. Arq Gastroenterol 2006;43.

36. Samulski DM, Noce F. Atividade física, saúde e qualidade de vida. In: Samulski DM, Organizador. Psicologia do Esporte: Manual para a Educação Física, Psicologia e Fisioterapia. São Paulo: Manole, 2002;301-18.

37. Rabelo DF, Néri AL. Recursos psicológicos e ajustamento pessoal frente à incapacidade funcional na velhice. Psicol Estud 2005;10:403.

38. Paskulin LMG, Molzahn A. Quality of life of older adults in Canada and Brazil. West J Nurs Res 2007;29:10-35.

39. Yeung, RR. The acute effects of exercise on mood state. Journal of Psychosomatic Research 1996;40:123-41. 